\title{
The Effects of Hypercapnia on DTI Quantification in Anesthetized Rat Brain
}

\author{
Abby Y. Ding, Edward S. Hui and Ed X. Wu*
}

\begin{abstract}
Diffusion Tensor Imaging (DTI) offers a valuable in vivo tool to characterize water diffusion behavior in biological tissues, particularly brain tissues. The accuracy of DTI derived parameters can directly affect the interpretation of underlying microstructures, physiology or pathologies. It is anticipated that measurement of apparent diffusion coefficient (ADC) using DTI could be influenced and complicated by the presence of water molecules in brain vasculature. However, little is known about to what degree does blood signal from vasculature affect the diffusion quantitation. In this study, we examined the effects of hypercapnia on DTI quantification in rat brains using inhalation of $5 \%$ carbon dioxide $\left(\mathrm{CO}_{2}\right)$. It was found that statistically significant changes occurred in parametric DTI maps in response to cerebrovascular challenges, indicating that vascular factors could interfere with in vivo DTI characterization of neural tissues. Consequently, hemodynamic alterations can potentially affect the DTI quantitation and detection of tissue microstructures and pathological alterations. Therefore, cautions must be taken when interpreting DTI parameters in vivo.
\end{abstract}

Index Terms-Cerebral vascular, Diffusivity Quantification, DTI, Hypercapnia

\section{INTRODUCTION}

$\mathrm{D}$ iffusion tensor imaging (DTI), which is sensitive to evaluate biological structure by calculating water diffusion coefficient[1-4], has been widely used for in vivo experiments with brain and heart tissues in normal and pathological states[5-16]. To such principle, it can be predicted that quantification of DTI parameters might be interfered by the presence of cerebral vasculature which would contaminate the diffusion signals from cellular fluid as well as other pathology-derived diffusivity changes [17-21]. However, the degree of blood signal from vasculature affects diffusivity has not been verified yet. In this study, we employed a hypercapnia rat model which would lead to passive dilation of cerebral blood vessels and increase cerebral blood flow by inhaling $5 \%$ carbon dioxide $\left(\mathrm{CO}_{2}\right)$ [17].

Hypercapnia, which can be caused by inhaling excessive concentrated Carbon Dioxide, is an efficient way to modulate cerebral blood flow (CBF) and blood volume (CBV). It also induces vasodilatory effects in brain, therefore is widely used to study the hemodynamic alteration in the absence of

Manuscript received April 7, 2009

Ed X. Wu, Abby Y. Ding and Edward S. Hui are with the Laboratory of Biomedical Imaging and Signal Processing and the Department of Electrical and Electronic Engineering, The University of Hong Kong, Pokfulam, Hong Kong (phone: (852) 2819-9713; e-mail: ewu@eee.hku.hk). neuronal activation. Thus, it is an important model of functional MRI (fMRI) studies for estimating vascular coupling [21].

The effects of hypercapnia on DTI quantification were evaluated by performing In vivo DTI experiments. Cerebrovascular response was confirmed by Blood Oxygenation Level Dependent (BOLD) [18] effect and physiological parameter monitoring. Thus, the correlation between blood flow and diffusivity values was investigated to provide substantial information on applying in vivo DTI for probing tissue microstructures as well as exogenic vascular regulations in brain.

\section{MATERIALS AND METHODS}

\section{A. Animal Preparation}

Normal adult female Sprague-Dawley rats (350-400g, N=2) were anesthetized with isoflurane/air using 3\% for induction and $1.9 \%$ for maintenance. Body temperature was maintained by circulating warm water in a heating pad. Continuous monitoring of respiration rate, heart rate, arterial oxygen saturation and rectal temperature were performed by animal probe (SA-Instruments, Stony Brook, NY) and maintained within normal physiological ranges. The monitoring data were exported for further $\mathrm{CO}_{2}$ activation analysis.

\section{B. Diffusion Tensor Imaging}

In vivo experiments were performed using a $7 \mathrm{~T}$ Bruker scanner. Each experiment included 48 continuous DTI trials, which were segmented into 6 couples of $\mathrm{OFF} / \mathrm{ON}$ periods by manually

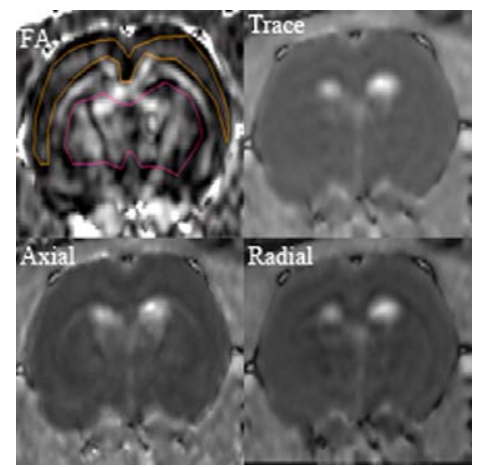

Fig.1. Cortex (orange) and subcortex (pink) regions of interest outlined on an FA map in a representative slice. Corresponded trace, axial and radial maps are also shown.

to the animal mouth cone at certain time points. Each couple of OFF/ON period included 5 DTI trials for normocapnia (OFF) with pure air inhalation and 3 DTI trials for hypercapnia $(\mathrm{ON})$ with $5 \% \mathrm{CO}_{2}$ /air inhalation. Diffusion-weighted (DW) images were acquired with a spin echo 2-shot EPI readout sequence with encoding scheme of 6 gradient directions. 5 additional images with $b$-value $=0(\mathrm{~B} 0$ images) were also acquired [22, 23]. The imaging parameters were: $\mathrm{TR} / \mathrm{TE}=3700 / 29.95 \mathrm{~ms}, \delta / \Delta=5 / 17 \mathrm{~ms}, \mathrm{FOV}=5 \times 5 \mathrm{~cm}^{2}$, 
acq matrix $=96 \times 96$, slice thickness $=1 \mathrm{~mm}(0.5 \mathrm{~mm}$ gap $)$, b-value of $1000 \mathrm{~s} / \mathrm{mm}^{2}$ and $\mathrm{NEX}=1$.

\section{Data Analysis}

DTI images were processed with DTI-studio v2.40 (http://cmrm.med.jhmi.edu, Johns Hopkins University, Baltimore, MD) to obtain trace, axial diffusivity, radial diffusivity, and FA maps for each single DTI trial respectively. Two regions of interest (ROIs) on cortex and subcortex region were manually drawn (Fig.1) based on FA maps using ImageJ software $\mathrm{v1.38x}$. When averaging the DTI derived values for normocapnia and hypercapnia conditions, the first 3 trials of each OFF period (about 4 minutes 30 seconds) were considered to be recovery time, and the first trial of each ON period (about 1 minute 30 seconds) were consider to be activation rise time[18, 21]. Therefore 2 trials of OFF periods and 2 trials of ON periods were used to evaluate the signal changes between normocapnia and hypercapnia. Voxel-based two-sample t-test analysis was performed on trace and B0 with threshold of $\mathrm{p}<0.05$ using SPM5 (FIL, UCL, London, UK).

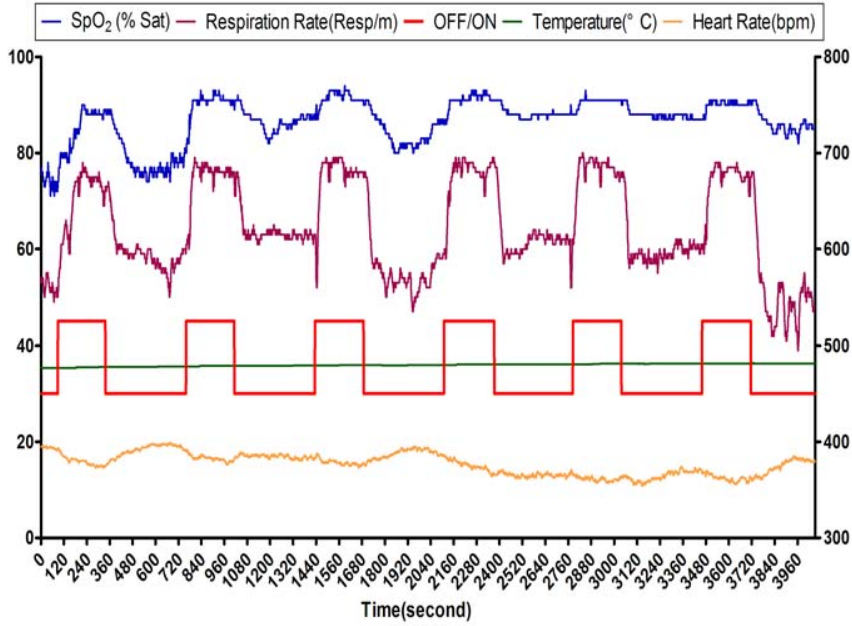

Fig.2. Continuous physiology records of heart rate, respiration rate, rectal temperature, and $\mathrm{SpO}_{2}$ during six couples of $\mathrm{OFF} / \mathrm{ON}$ periods.
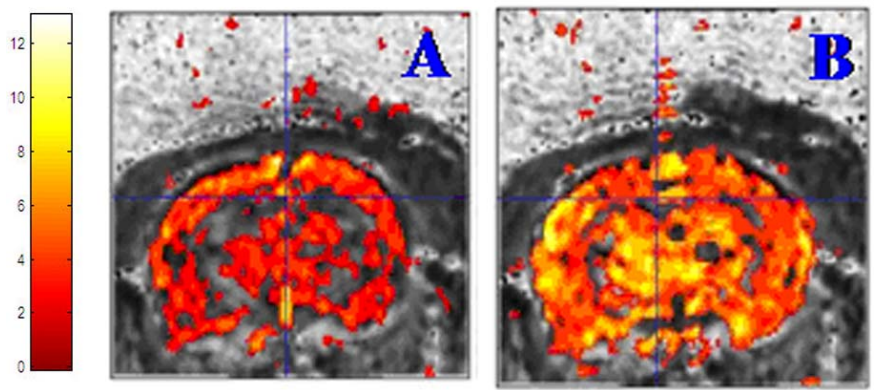

Fig.3. Statistical map overlaid on FA map of an animal. Voxel-based two-sample t-test was perform respectively on trace and B0 from 6 repeats of normocapnia $(\mathrm{OFF})$ and hypercapnia $(\mathrm{ON})$ with threshold of $\mathrm{p}<0.05$.

\section{RESULTS}

Fig.2. shows the physiology parameters during an experiment. Respiration rate rose from $59 \pm 6 \mathrm{resp} / \mathrm{m}$ during OFF period to $74 \pm 5 \mathrm{resp} / \mathrm{m}$ during ON period which revealed cerebrovascular response to hypercapnic challenge [19] while other physiology parameters were consistent with previous studies in rats under hypercapnia $[18,19]$.

Fig.3B depicts the statistical significant changes in B0 signal intensity which can be considered to be BOLD effect between all OFF/ON periods in a representative slice. Fig.3A shows the statistical significance of trace value changes between all OFF/ON periods. It's obvious that both $\mathrm{B} 0$ and trace values have significantly changed on the majority of the brain especially in cortex and subcortex area, which suggested that typical regions might be more susceptible to hypercapnic challenge.

Changes in average trace values during normocapnia and hypercapnia conditions in cortex and subcortex regions are illustrated in Fig. 4 . The average increase percentage is $1.6 \%$ in cortex and $1.7 \%$ in subcortex among trials and animals. Continuous 48 axial, radial, mean diffusivity and FA values in cortical area were plotted versus time as shown in Fig.5. There are coordinate fluctuation profiles in mean diffusivity, axial and radial values as normocapnia/hypercapnia conditions changed. Reasonable activation rising slopes after switching on $\mathrm{CO}_{2}$ inhalation as well as descending slopes after switching off $\mathrm{CO}_{2}$ inhalation can also be observed [21].

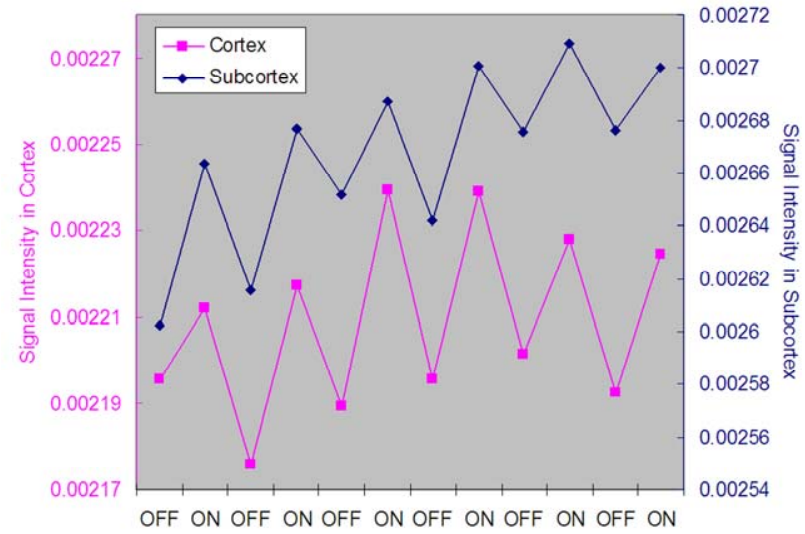

Fig.4. Average trace value (in $\mathrm{mm}^{2} / \mathrm{s}$ ) of ROIs versus 6 repeats of $\mathrm{OFF} / \mathrm{ON}$ trials.

\section{DisCUSSIONS}

The statistical dependence of $\mathrm{B} 0$ value between normoxia/hypercapnia has shown strong evidence of BOLD effect which indicated the elevation of both CBV and CBF caused by hemodynamic activation under hypercapnic challenge[18]. Furthermore, the statistical significance in trace value demonstrated that diffusivity quantification increased accordingly correspond with the $\mathrm{CO}_{2}$ inhalation. These quantification alterations are probably caused by the cerebral vascular smooth muscle response to the increased partial pressure of $\mathrm{CO}_{2}$ in blood[18], as a result of which would enhance the ion exchange in local acidic environment. 


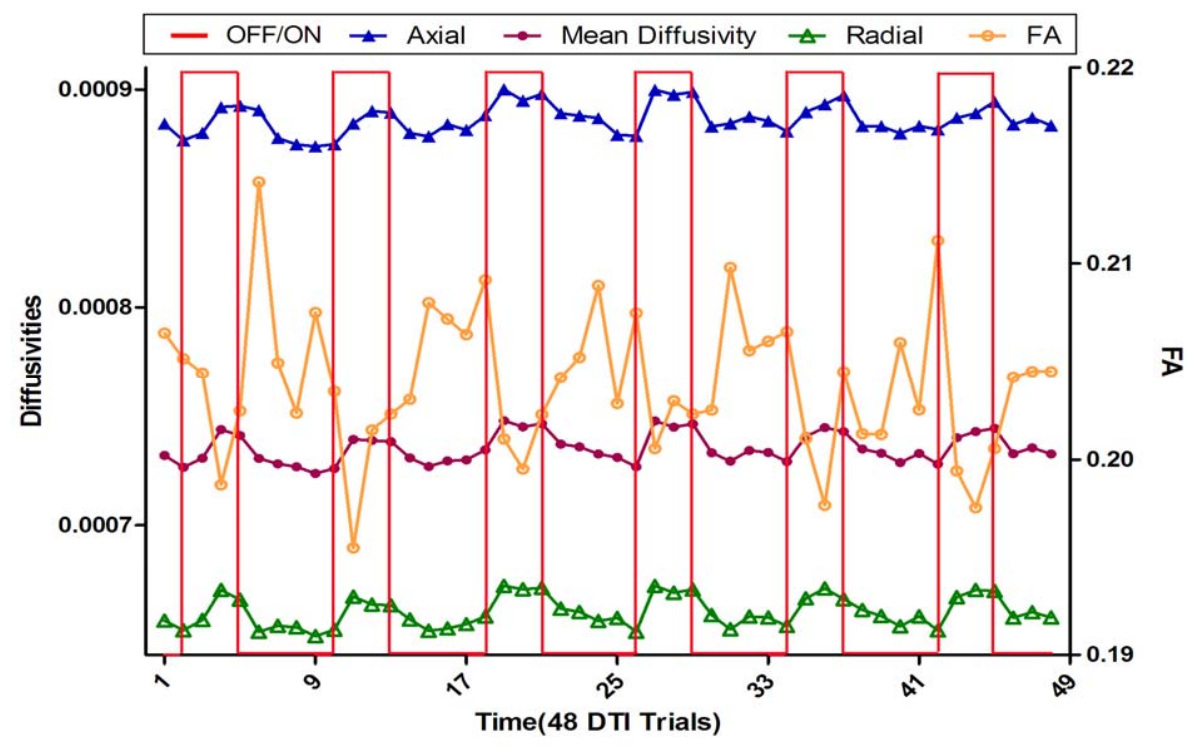

Fig.5. Axial, radial, mean diffusivity (in $\mathrm{mm}^{2} / \mathrm{s}$ ) and FA values in cortex versus 48 continuous DTI trials, each DTI trial lasted about 1 minute 25 seconds.

In addition, it is likely that grey matter, which is known to be rich in vessels, diffusivity changes are significantly higher than other regions with respect to this cerebrovascular reactivity. ROI measurements further verified that in these typical regions, the signal change in DTI quantifications were solid.

Meanwhile, a pattern corresponds with mean diffusivity fluctuation could be found in axial and radial values, which were also shifted up and down consistent with OFF/ON periods. These might be contributed by the CBF and CBV alterations during hypercapnia, when excessive $\mathrm{CO}_{2}$ was hydrated to local acidic environment and resulted in a passive vessel dilation as well as decreased resistance of cerebral arterial smooth muscle[18]. FA value may also be characterized by that a deepest point arose right after switching on $\mathrm{CO}_{2}$ inhalation then gradually climbed up to a peak following turning-off $\mathrm{CO}_{2}$ inhalation. These may suggest that hemodynamic activation had led to an enhanced effect on macro vessels in certain directions in brain.

\section{CONCLUSION}

As observed from this in vivo study, statistically significant changes occur in parametric DTI maps in response to cerebrovascular challenges, indicating that vascular factors can interfere with in vivo DTI characterization of neural tissues. Consequently, hemodynamic alterations can potentially affect the DTI quantification and detection of tissue microstructures and pathological alterations. Therefore, cautions must be taken when interpreting DTI parameters in vivo.

\section{REFERENCES}

[1] D. Le Bihan, "Molecular diffusion nuclear magnetic resonance imaging," Magn Reson Q, vol. 7, pp. 1-30, Jan 1991.
S. Mori and P. B. Barker, "Diffusion magnetic resonance imaging: its principle and applications," Anat Rec, vol. 257, pp. 102-9, Jun 151999.

M. A. Horsfield and D. K. Jones, "Applications of diffusion-weighted and diffusion tensor MRI to white matter diseases - a review," NMR Biomed, vol. 15, pp. 570-7, Nov-Dec 2002.

S. Mori and J. Zhang, "Principles of diffusion tensor imaging and its applications to basic neuroscience research," Neuron, vol. 51, pp. 527-39, Sep 72006.

Y. Wang, P. T. Cheung, G. X. Shen, E. X. Wu, G. Cao, I. Bart, W. H. Wong, and P. L. Khong, "Hypoxic-ischemic brain injury in the neonatal rat model: relationship between lesion size at early MR imaging and irreversible infarction," AJNR Am J Neuroradiol, vol. 27, pp. 51-4, Jan 2006.

E. X. Wu, Y. Wu, J. M. Nicholls, J. Wang, S. Liao, S. Zhu, C. P. Lau, and H. F. Tse, "MR diffusion tensor imaging study of postinfarct myocardium structural remodeling in a porcine model," Magn Reson Med, vol. 58, pp. 687-95, Oct 2007.

E. X. Wu, Y. Wu, H. Tang, J. Wang, J. Yang, M. C. Ng, E. S. Yang, C. W. Chan, S. Zhu, C. P. Lau, and H. F. Tse, "Study of myocardial fiber pathway using magnetic resonance diffusion tensor imaging," Magn Reson Imaging, vol. 25, pp. 1048-57, Sep 2007.

Y. Wang, P. T. Cheung, G. X. Shen, I. Bhatia, E. X. Wu, D. Qiu, and P. L. Khong, "Comparing diffusion-weighted and T2-weighted MR imaging for the quantification of infarct size in a neonatal rat hypoxic-ischemic model at $24 \mathrm{~h}$ post-injury," Int $J$ Dev Neurosci, vol. 25, pp. 1-5, Feb 2007.

E. S. Hui, M. M. Cheung, L. Qi, and E. X. Wu, "Towards better MR characterization of neural tissues using directional diffusion kurtosis analysis," Neuroimage, vol. 42, pp. 122-34, Aug 12008. S. Wang, E. X. Wu, C. N. Tam, H. F. Lau, P. T. Cheung, and P. L. Khong, "Characterization of white matter injury in a hypoxic-ischemic neonatal rat model by diffusion tensor MRI," Stroke, vol. 39, pp. 2348-53, Aug 2008.

S. Wang, E. X. Wu, D. Qiu, L. H. Leung, H. F. Lau, and P. L. Khong, "Longitudinal diffusion tensor magnetic resonance imaging study of radiation-induced white matter damage in a rat model," Cancer Res, vol. 69, pp. 1190-8, Feb 12009.

Y. Wu, C. W. Chan, J. M. Nicholls, S. Liao, H. F. Tse, and E. X. $\mathrm{Wu}$, "MR study of the effect of infarct size and location on left ventricular functional and microstructural alterations in porcine models," J Magn Reson Imaging, vol. 29, pp. 305-12, Feb 2009.

M. M. Cheung, E. S. Hui, K. C. Chan, J. A. Helpern, L. Qi, and E. $\mathrm{X}$. Wu, "Does diffusion kurtosis imaging lead to better neural 
tissue characterization? A rodent brain maturation study," Neuroimage, vol. 45, pp. 386-92, Apr 12009.

[14] K. C. Chan, P. L. Khong, M. M. Cheung, S. Wang, K. X. Cai, and E. X. Wu, "MRI of late microstructural and metabolic alterations in radiation-induced brain injuries," J Magn Reson Imaging, vol. 29, pp. 1013-20, May 2009.

[15] Y. Wu and E. X. Wu, "MR study of postnatal development of myocardial structure and left ventricular function," J Magn Reson Imaging, May 222009.

[16] K. C. Chan, P. L. Khong, H. F. Lau, P. T. Cheung, and E. X. Wu, "Late Measures of Microstructural Alterations in Severe Neonatal Hypoxic-Ischemic Encephalopathy by MR Diffusion Tensor Imaging," Int J Dev Neurosci, Jun 52009.

[17] L. L. Latour and S. Warach, "Cerebral spinal fluid contamination of the measurement of the apparent diffusion coefficient of water in acute stroke," Magn Reson Med, vol. 48, pp. 478-86, Sep 2002.

[18] M. E. Brevard, T. Q. Duong, J. A. King, and C. F. Ferris, "Changes in MRI signal intensity during hypercapnic challenge under conscious and anesthetized conditions," Magn Reson Imaging, vol. 21, pp. 995-1001, Nov 2003.

[19] K. Sicard, Q. Shen, M. E. Brevard, R. Sullivan, C. F. Ferris, J. A King, and T. Q. Duong, "Regional cerebral blood flow and BOLD responses in conscious and anesthetized rats under basal and hypercapnic conditions: implications for functional MRI studies," J Cereb Blood Flow Metab, vol. 23, pp. 472-81, Apr 2003.

[20] A. Schafer, S. Zysset, W. Heinke, and H. E. Moller, "Hypercapnia-induced effects on image contrast based on intermolecular double-quantum coherences," Magn Reson Med, vol. 60, pp. 1306-1312, Nov 242008.

[21] J. Lu, G. Dai, Y. Egi, S. Huang, S. J. Kwon, E. H. Lo, and Y. R. $\mathrm{Kim}$, "Characterization of cerebrovascular responses to hyperoxia and hypercapnia using MRI in rat," Neuroimage, vol. 45, pp. 1126-1134, May 12009

[22] D. K. Jones, M. A. Horsfield, and A. Simmons, "Optimal strategies for measuring diffusion in anisotropic systems by magnetic resonance imaging," Magn Reson Med, vol. 42, pp. 515-25, Sep 1999.

[23] S. Skare, M. Hedehus, M. E. Moseley, and T. Q. Li, "Condition number as a measure of noise performance of diffusion tensor data acquisition schemes with MRI," J Magn Reson, vol. 147, pp. $340-52$, Dec 2000 